\title{
Study on Methods for Improving LMD End Effect by Gaussian Process Based on the Particle Swarm Optimization Algorithm
}

\author{
Wang Fengwei ${ }^{1,2, a}$, Luo Yiyong ${ }^{1}$, Chi Qicai ${ }^{1}$, Zhou Yunqi $^{3}$, \\ Zhou Shijian ${ }^{4, b}$, \\ ${ }^{1}$ School of Surveying and Mapping Engineering, East China University of Technology, Nanchang \\ 330013, China \\ ${ }^{2}$ Key Laboratory of Watershed Ecology and Geographical Environment Monitoring, \\ National Administration of Surveying, mapping and Geoinformation, Nanchang 330013, China \\ ${ }^{3}$ The Hong Kong Polytechnic University, Department of Land Surveying and Geo-Informatics, \\ HongKong, China \\ ${ }^{4}$ Nanchang Hangkong University, Nanchang 330063, China \\ a1175577628@qq.com, b408608628@qq.com
}

Keywords: Local mean decomposition, End effect, Particle swarm optimization, Gaussian process, Mechanical vibration signal

\begin{abstract}
The LMD is a new method for analyzing non-stationary signals. It can decompose complicated signals into a set of single-component signals, each of which has physical sense. However, performing the LMD will produce end effects which make results distorted. After analyzing the reasons for these, the article takes advantage of the Gaussian process algorithm to overcome the end effects of LMD. To improve the precision of GP algorithm of endpoint extension, the authors use the particle swarm algorithm to optimization the GP hyper parameter and select the optimal covariance function. Experimental results showed that the GP algorithm of particle swarm optimization (PSO) can predict the two ends of the data signal more accurately, improve the accuracy of LMD and avoid the adverse effects caused by end effect according to the internal characteristics of the signal. Therefore the PSO-GP algorithm is a better method to improve the end effect.
\end{abstract}

\section{Introduction}

The LMD method possesses various good properties about theories, which makes it to be suitable for handling non-stationary signals. However, it is a new signal handling method, which needs more improvement when applying in practical situations. The end effect is one of the obvious and urgent problems which needed to be deal with [1]. Just as the empirical mode decomposition (EMD) [2-7], LMD also has the similar process to decompose complicated signals into a set of single-component signals according to local extreme signals continuously. During the decomposition process, it is needed to smooth continuous from the local extreme function lines which is made up by local extreme signal points and envelope estimation function lines. However, due to the fact that the two ends of signals may be neither a local maximum nor a local minimum, it could lead to the two end signals' divergence phenomenon of local extreme function lines and envelope estimation function lines during the smoothing process, in addition, this divergence result would pollute the entire data sequence gradually and inwardly, which would give rise to serious distortion of decomposition results.

In terms of the end effect problem of EMD method, there are various improvement methods which have achieve certain goals, however existing limitations in the meanwhile. For example, extreme signals extension method [8] only take into account information of some extreme signals which are close to end signals without conendration of internal characteristics of the signals. As for tackling complicated non-stationary signals, these results are less satisfactory. The boundary wave matching algorithm method [9] conendr internal characteristics and tendency of the signals, 
however, this method lack adaptivity and have poor results about mutational signals of boundary waves. As for neural networks [10] and various prediction extension methods, the improvement effects are largely dependent on the setting parameters of prediction methods, simultaneously, the computation time is longer and this methods lack of practicality. Gaussian process (Gaussian Process, GP) is a recently developed new machine learning technique. The Gaussian process has rigorous theoretical statistical learning theories and foundations, which possess good adaptability to deal with complex problems of high dimensionality, sample timber and nonlinearity and so on, and obtain prediction accuracy and probability scientifically, which is a preferred non linear learning method [11, 12]. Based on experience to improve the end effect of EMD, this article conduct detailed analysis of the reasons leading to LMD end effect, as well as conendr applying Gaussian process to conduct end signal extension of Local Mean Decomposition. In order to improve the accuracy of prediction model of GP algorithm, it is needed to select suitable optimal covariance function for engineering cases and utilize the particle swarm algorithm to optimization the GP hyper parameter. Through simulation signals and practical engineering signals, the results of methods and algorithms this article has discussed about to inhibit LMD Decomposition end effect could be validated.

\section{Local Mean Decomposition and End Effect}

Local mean decomposition method is a new adaptive time-frequency analysis method. It can adaptively decompose complex multi-component signals into the collection of finite number of mode functions, where each component PF is actually an amplitude modulation of single component--frequency modulated (FM) signal, which is able to extract instantaneous amplitude and instantaneous frequency directly. When all of the instantaneous amplitude and instantaneous frequency of PF component combinations, it is supposed to gain the complete frequency distribution of the original signals. The whole idea of LMD is based on the local time scale characteristic of the signals, and then to decompose signals from high frequency to low frequency according to hierarchy gradually. Every component after decomposition is stationary and possesses physical meaning. For an arbitrary signal $x(t)$, the decomposition process is as follows [13-16]:

(1) Find all local extreme signals $n_{i}$ of original signals, and then calculate the average values of adjacent low extreme signals

$$
m_{i}=\left(n_{i}+n_{i+1}\right) / 2
$$

Use straight lines to connect all adjacent average values $m_{i}$, and then apply average moving method to smooth to obtain local mean function $m_{11}(\mathrm{t})$.

(2) To obtain Envelope estimation: $a_{i}=\left|n_{i}-n_{i+1}\right| / 2$ Use straight lines to connect adjacent envelope estimations $a_{i}$, and then apply average moving method to smooth to obtain the envelope estimation function $a_{11}(\mathrm{t})$. Decompose the local mean function $m_{11}(\mathrm{t})$ from the original signals $\mathrm{x}(\mathrm{t})$ and then obtain:

$$
h_{11}(t)=x(t)-m_{11}(t)
$$

(3) Utilize $h_{11}(t)$ dividing envelope estimation function $a_{11}(\mathrm{t})$ which aim to conduct mediation on $h_{11}(t)$, this step obtains : $s_{11}(t)=h_{11}(t) / a_{11}(t)$,and then repeat the above steps is able to about the envelope estimation function $\mathrm{a}_{12}(t)$ of $h_{11}(t)$, if $\mathrm{a}_{12}(t)$ not equal to 1 , which illustrates that $s_{11}(t)$ is not a pure frequency modulated (FM) signal, then it is needed to repeat the iterative process $n$ times until such time as a pure frequency modulated (FM) signal $s_{1 n}(t)$ appears. It means that the $s_{1 n}(t)$ 's envelope estimation function $\mathrm{a}_{1(\mathrm{n}+1)}(t)=1$.

(4) Multiple all envelope estimation functions during the iterative process to obtain envelope 
signal (instantaneous amplitude function)

$\mathrm{a}_{1}(t)=a_{11}(t) a_{12}(t) \cdots a_{1 n}(t)=\prod_{q=1}^{n} a_{1 q}(t)$

(5) Multiple the envelope signal $a_{1}(t)$ and pure frequency modulated (FM) signal $\mathrm{s}_{1 n}(t)$, it is able to obtain the first PF component of original signals, $P_{1}(t)=a_{1}(t) \mathrm{s}_{1 n}(t)$, which contains the highest frequency component of original signals, and it is a single component amplitude-amplitude modulated (AM) signal. Its instantaneous amplitude is the envelope signal $a_{1}(t)$, in addition, its instantaneous frequency $f_{1}(t)$ could be determined from the pure frequency modulated (FM) signal.

(6) Separate the first PF component $p_{1}(t)$ from the original signals $x(t)$ out to gain a new signal $u_{1}(t)$, regard $u_{1}(t)$ as raw data and then repeat above steps, k cycle times, until $u_{k}$ is a monotonic function appearing.

$$
\left\{\begin{array}{c}
u_{1}(t)=x(t)-p_{1}(t) \\
u_{2}(t)=x(t)-p_{2}(t) \\
\vdots \\
u_{k}(t)=x(t)-p_{k}(t)
\end{array}\right.
$$

(7) The original signals $x(t)$ can be reconstructed by all PF components and $u_{k}$

$$
x(t)=\sum_{p=1}^{k} p_{p}(t)+u_{k}(t)
$$

In the formula (5), if we recombinant all instantaneous frequency and instantaneous amplitude of all PF components according to the time-- frequency - three-dimensional distribution, it is able to obtain the complete time and frequency distribution of the original signals based on LMD decomposition.

Derived from the above decomposition process of LMD, it is found that it would lead to end effects. The key reason is that information of local extreme points are needed to obtain local extreme function and envelope estimation function, in which the local extreme function is gained from the straight lines which are connected by average values of adjacent extreme points during continuous smoothing process. The envelope estimation function is obtained from straight lines which are connected by absolute difference adjacent extreme points of during continuous smoothing process. However, the two end points of data may not necessarily be the extreme signals, therefore the resulting local average curve and the envelope estimation curve being at both ends of the data is unreasonable.

\section{The End Points Extension based on Gaussian Process}

Gaussian Process. Gaussian process (Gaussian Process, GP) is a recently developed new machine learning technique. The Gaussian process has rigorous theoretical statistical learning theories and foundations, which possess good adaptability to deal with complex problems of high dimensionality, sample timber and nonlinearity and so on, and obtain prediction accuracy and probability scientifically, which is a preferred non linear learning method [11, 12]. The statistical characteristics of Gaussian Process depend on its mean function and covariance function. The conendrable regression model[17-19]:

$$
y=f(x)+\varepsilon
$$

In formula (6), $x$ is input vector, $y$ is observation, noise $\varepsilon \square N\left(0, \delta_{n}^{2}\right)$. According to Bayesian 
posterior probability formula, it is able to get the predicted value expectation and variance of $y^{\prime}$ respectively:

$$
\begin{aligned}
& \mu_{y^{\prime}}=C\left(x^{\prime}, X\right)\left[C(X, X)+\delta_{n}^{2} I_{n}\right]^{-1} y \\
& \delta_{y^{\prime}}^{2}=C\left(x^{\prime}, x^{\prime}\right)-C\left(x^{\prime}, X\right)\left[C(X, X)+\delta_{n}^{2} I_{n}\right]^{-1} C\left(X, x^{\prime}\right)
\end{aligned}
$$

In Gaussian Process, covariance function Gaussian process is equivalent to the kernel function commonly used covariance function is index squared covariance, which is:

$$
C\left(x_{i}, x_{j}\right)=\delta_{f}^{2} \exp \left[-\frac{1}{2}\left(x_{i}-x_{j}\right)^{T} M\left(x_{i}-x_{j}\right)\right]+\delta_{n}^{2} \delta^{i j}
$$

In formula (9), $\delta_{f}^{2}$ is the signal variance of kernel function; $M=\operatorname{diag}\left(l^{-2}\right)$ is the diagonal matrix of hyper parameter, $l$ is the variance scale; and $\delta^{i j}$ is the Kronecker symbol.

Let $\theta=\left\{M, \delta_{f}^{2}, \delta_{n}^{3}\right\}$ as hyper parameter. The optimal parameters are generally obtained by super-logarithmic maximum likelihood method. Like negative log-likelihood function and hyper parameter $\theta$ as follows:

$$
\begin{aligned}
& L(\theta)=\frac{1}{2} y^{T} C^{-1} y+\frac{1}{2} \log |C|+\frac{n}{2} \log 2 \pi \\
& \frac{\partial L(\theta)}{\partial \theta_{i}}=\frac{1}{2} \operatorname{tr}\left[\left(\alpha \alpha^{T}-C^{-1}\right) \frac{\partial C}{\partial \theta_{i}}\right]
\end{aligned}
$$

In formula, $\alpha=C^{-1} y$.

Particle Swarm Algorithm to Optimization the GP Hyper Parameters. Particle Swarm Optimization (PSO) is a field of computational intelligence, in addition to the ant colony algorithm, the algorithm of a fish swarm intelligence optimization algorithm. PSO algorithm firstly initializes a group of particles in the feasible solution space, and each particle represents a potential optimal extremal optimization problem with the three indicators of location, speed and fitness value to represent the particle characteristics. Adaptation value is calculated by adaptation function, which representing the quality of particles. Particles move in the solution space. By tracking Pbest and Gbest, it is able to update individual position groups. Pbest refers to the calculated best position for adaptation among all locations which individual experienced, while Gbest refers to the optimum position for adaptation for the all particles. Every time particle updated every position, an adaptation value would be calculated. It is able to compare the adaptation value, Pbest and Gbest of new particle to update the new locations of Pbest and Gbest.

Suppose in a $D$ dimension search space, the population composed by $n$ particles $X=\left(X_{1}, X_{2}, \cdots, X_{n}\right)$, wherein the number $i$ particle is represented as a $D$ dimension vector $X_{i}=\left(x_{i 1}, x_{i 2}, \cdots, X_{i D}\right)^{\mathrm{T}}$, representing the position of the numberiparticle in $D$ dimensional search space, also represents a potential solution to the problem. Based on objective function it is able to calculate the corresponding adaptation value of each particle location $X_{i}$. The velocity of number $i$ particle is $V_{i}=\left(V_{i 1}, V_{i 2}, \cdots, V_{i D}\right)^{\mathrm{T}}$, the Pbest is $P_{i}=\left(P_{i 1}, P_{i 2}, \cdots, P_{i D}\right)^{\mathrm{T}}$ and Gbest is $P_{g}=\left(P_{g 1}, P_{g 2}, \cdots, P_{g D}\right)^{\mathrm{T}}$.

In each iteration process, the particles utilize Pbest and Gbest to update its velocity and position, which are 


$$
\begin{aligned}
& V_{i d}^{k+1}=\omega V_{i d}^{k}+c_{1} r_{1}\left(P_{i d}^{k}-X_{i d}^{k}\right)+c_{2} r_{2}\left(P_{g d}^{k}-X_{g d}^{k}\right) \\
& X_{i d}^{k+1}=X_{i d}^{k}+V_{i d}^{k+1}
\end{aligned}
$$

where, $\omega$ is the inertia weight; $d=1,2, \cdots, D ; i=1,2, \cdots, n ; k$ is the current number of iteration; $V_{i d}$ is the velocity of the particles; $c_{1}$ and $c_{2}$ are non-negative constants, becoming acceleration factor; $r_{1}$ and $r_{2}$ are random numbers which are distributed in the range of [0,1].

For a Gaussian process, the parameters of kernel functions and likelihood function play a decisive role in Gaussian process modeling learning. Gaussian processes using traditional conjugate gradient method to search parameters, whereas it present high dependence on the initial value, the iteration number is difficult to determine, in addition, it is easy to access local optima. Therefore, the authors used PSO method to optimize the parameter of Gaussian process' kernel function, then to obtain PSO-GP algorithm, concrete steps are as follows [24-26]:

1) Based on observational data sample, the data is divided into a training set and test set;

2) Do Initial settings. To determine the size of the particles, the number of iterations, the initial velocity of the particles and particles' initial position, each particles vector corresponds to a sequence parameter, namely the parameters of the kernel function. Different kernel functions correspond to different hyper parameters. Regard NN kernel function as example, $x=[l, s f]$.

3) Compared model with sample to conduct training and learning, then to calculate the adaptation value of each particle. The adaptation values are derived from the adaptation functions. If defines

$$
\min _{l, s f} \frac{1}{n} \sqrt{\sum_{i=1}^{n}\left(y_{i}-\overline{y_{i}}\right)^{2}}
$$

In formula, $y_{i}$ is the true value; $\bar{y}_{i}$ is the predicted value; $n$ is the number of samples;

4) According to current hyper parameters $l$ and $s f$, it is able to establish GP model to calculate the adaptation value through the formula (14);

5) Memorize corresponding Pbest and Gbest of individual and group's optimal adaptation value;

6) Based on formula (12) and (13) to update the particles' velocity and position, and search for better $l$ and $s f$;

7) Harness hyper parameters obtained from the use of PSO optimization on test samples, and then to gain the prediction value of signal sequence extreme points.

Data Extension Method Based on PSO-GP. According to the two main effective factors of LMD end effects: extreme points and the distances between them. Extracted from original data to obtain maximum point, minimum point and the distance between the extreme points respectively, and then to divide to three different group sequence $J_{d_{s}} J x, D$. Set them separately as:

$$
\begin{aligned}
& J_{d}=\left(J_{d 1}, J_{d 2}, J_{d 3}, \cdots, J_{d n}\right) \\
& J_{x}=\left(J_{x 1}, J_{x 2}, J_{x 3}, \cdots, J_{x n}\right) \\
& D=\left(D_{1}, D_{2}, D_{3}, \cdots, D_{n}\right)
\end{aligned}
$$

Firstly we could use PSO-GP model to conduct extension to the right end of original data, which is sequences' prediction based on PSO-GP model formula (15). Each sequence only need three predictive values and then combines these three sequences predicted values together, thus making the extension to right end of the original data.

Conduct reverse process on formula (15) and results are shown as the formula (16):

$$
J_{d}^{-1}=\left(J_{d n}, J_{d(n-1)}, J_{d(n-2)}, \cdots, J_{d 1}\right)
$$




$$
\begin{aligned}
& J_{x}^{-1}=\left(J_{x n}, J_{x(n-1)}, J_{x(n-2)}, \cdots, J_{x 1}\right) \\
& D^{-1}=\left(D_{n}, D_{(n-1)}, D_{(n-2)}, \cdots, D_{1}\right)
\end{aligned}
$$

In a similar way, it is possible to conduct prediction on the left end of original data and then to proceed the extension to the left end. Finally combining together, we get the final extension data.

\section{The Simulation Signal Analysis}

In order to verify the feasibility of this method, we take three sine and cosine superimposed signal to analyze, the signal expression is $\mathrm{x}=\cos (2 *$ pi. $* 5 * \mathrm{t})+2 * \sin (2 *$ pi. $* 25 * \mathrm{t})+3 *$ $\cos (2 *$ pi. $* 100 * \mathrm{t}), \quad t \in[0,1]$.According to the trend and taking into account Gaussian process, it is able to select more suitable covariance function to conduct training. Select the part of extreme point sequences as training samples and select another part as the test samples, finally conduct extension from the left and right end. According to the LMD decomposition principle, only three maximum and minimum extension points from the left and right ends are needed.

Decomposing signal $\mathrm{x}(\mathrm{t})$ by LMD decomposition methods directly, the results are shown in Figure1, PF1-PF5 boundaries occurred serious distortions. From Hilbert spectrum (Fig. 2), it can be found that there are obvious distortions appearing in both two ends and edge divergence phenomenon presenting. Fig 3 and 4 are the results of endpoint extension's LMD decomposition and Hilbert spectrum through PSO-GP algorithms. Compared with decomposition results without endpoint extension, it is obvious that edge divergence phenomenon have improved a lot, the residue was significantly less than the amount of direct decomposition. To further verify the effect of the end effect to improve the situation, the article harnesses three indicators of LMD end effect to evaluate.

1) Energy aspect: the essence of the end effect is the envelope signal distortion, which produce a number of fraudulent, so that all PF component of the total energy will increase, therefore the use of energy exploded around to assess the impact of endpoint effects [28, 29].

$$
\theta=\frac{\left|\sqrt{\sum_{i=1}^{n} P F_{i}^{2}}-P F_{\text {orginal }}\right|}{P F_{\text {orginal }}}
$$

whereby, $P F_{\text {orginal }}$ represents the original effective signal values; $P F_{i}$ denotes a modal component of number $i ; n$ is the total number of $P F$ (including the residual components of LMD); the smaller the $\theta$ value, indicating less end effect, which means that better endpoint extension results.

2) Compare modal component from LMD decomposition and corresponding parameter $\rho$ of original signals to appraise the accuracy of $P F$.

$$
\rho_{i}\left(x_{i}(t), P F_{i}(t)\right)=\frac{\operatorname{cov}\left(x_{i}(t), P F_{i}(t)\right)}{\sqrt{\delta\left(x_{i}\right)} \sqrt{\delta\left(P F_{i}\right)}}
$$

In the formula, $\operatorname{cov}()$ represents covariance; $\delta()$ represents the variance; $P F_{i}$ denotes a modal component of number $i \mathrm{PF} ; x_{i}$ represents the original signal corresponding to the constituent components. The higher the $\rho$ value, indicating that the inhibitory effect end effect is better.

3) Compare different methods of computing timet (unit: s), to ensure the treatment effect in the same time, try to avoid overly complex algorithm. 


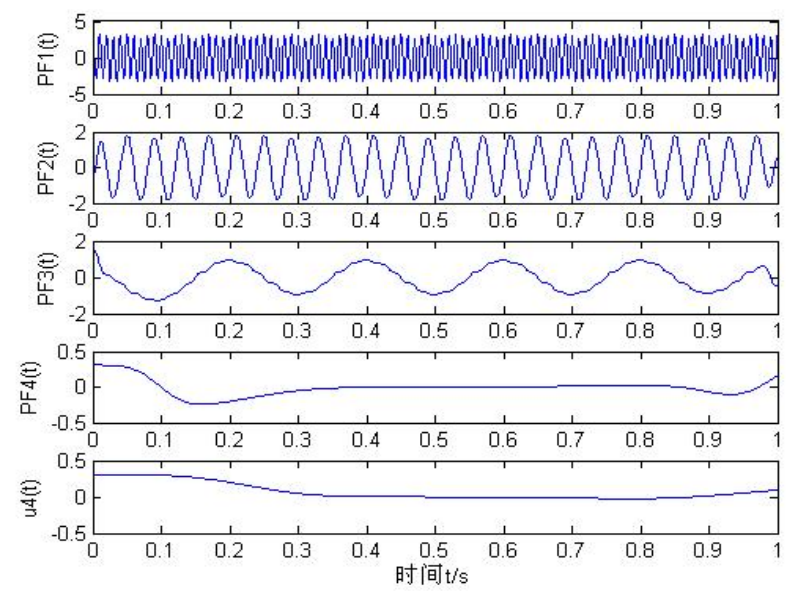

Fig. 1 Decomposition of LMD without extrension.

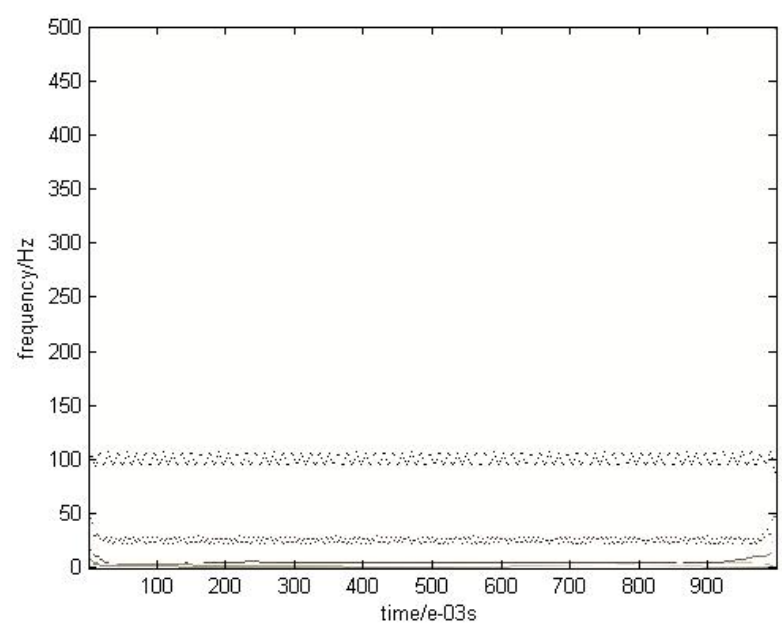

Fig. 2 Hilbert-huang spectrum without ertension.

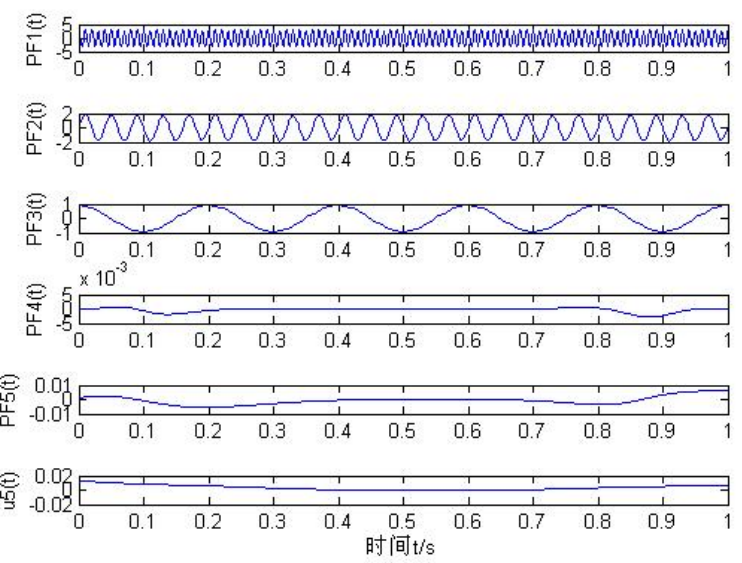

Fig. 3 Decomposition of ertension by PSO-GP algorithm. 


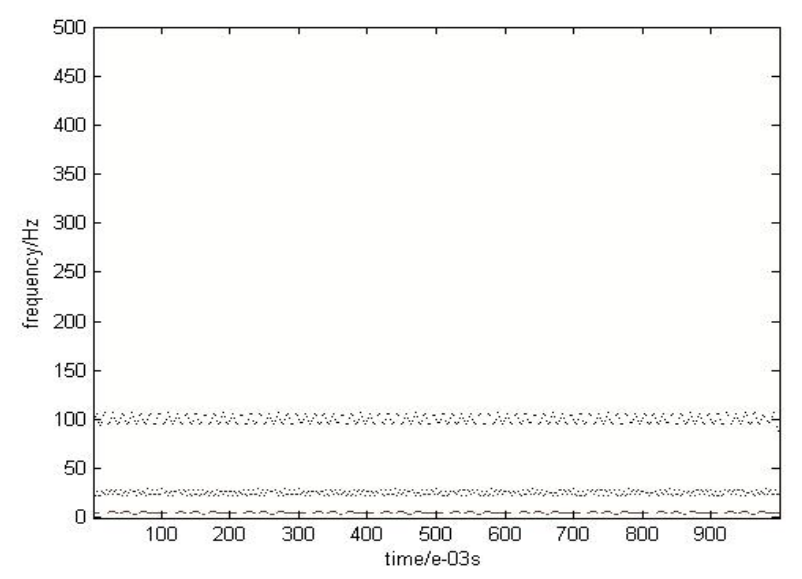

Fig. 4 Hilbert-huang spectrum by PSO-GP algorithm.

This article uses PSO Gaussian process method to conduct endpoint extension with using MATLAB program to proceed LMD local mean decomposition. Signal decomposition components are shown in Fig. 1 and Fig 3. It is found in the figure that the signal decompositions without end effect present noticeable swing. Fig. 3 and Fig. 4 adopt PSO-GP algorithm to conduct endpoint extension. From signal decomposition figure and Hilbert spectrum, it could be seen that there are nearly no swing on both ends of signal decompositions, as well as no fluctuation in Hilbert spectrum figure. To further verify the effect of the proposed algorithm's improving effect offend effect in the article, three types of evaluation index are used to evaluate, which could be seen in Table 1.

Table 1. Comparision of methods of end effect.

\begin{tabular}{cccc}
\hline Signal & Evaluation Index & Without extension & PSO-GP method \\
\hline \multirow{4}{*}{$\mathrm{x}(\mathrm{t})$} & $\rho_{P F_{1}}$ & 0.0644 & 0.0288 \\
& $\rho_{P F_{3}}$ & 0.9960 & 0.9962 \\
& $\rho_{P F_{3}}$ & 0.9923 & 0.9986 \\
& $t$ & 0.9496 & 0.9991 \\
& & 0.5294 & 2.1773 \\
\hline
\end{tabular}

\section{Mechanical Engineering Experiment and Results Analysis}

In reality, the mechanical failure signals are usually periodic, while partially have random features. Therefore, this paper investigates the antifriction bearing data of single point drive end failures and fan end failures collected by the US Case Western Reserve University laboratory. The failures have diameters ranging from 0.007 inch to 0.040 inch and occur at inner races, balls and outer races. The vibration data of testing power-driven machine records power load from 0 to 3 horsepower (1797 to $1720 \mathrm{rpm}$ ). The vibration signal is collected by a 16-channel DAT recorder, and processed with MATLAB. The author adopts covNNone nerve net function and meanZero mean function to conduct a trial forecast of extremum serial of the signals. Afterwards, LMD decomposition of the signals is conducted. The decomposition result is shown in Fig. 7-10, with the original signals shown in Fig. 6. The test parameters adopted is shown in Table 2.

Table 2. Experimental parameters.

\begin{tabular}{cccc}
\hline Bearing type & $\begin{array}{c}\text { Sampling } \\
\text { frequency/KHZ }\end{array}$ & $\begin{array}{c}\text { Rotate } \\
\text { speed(r.min) }\end{array}$ & $\begin{array}{c}\text { Fault } \\
\text { size } \\
\text { /mm }\end{array}$ \\
\hline $6205-2 \mathrm{RS}(\mathrm{SKF})$ & 48 & 1797 & 0.356 \\
\hline
\end{tabular}




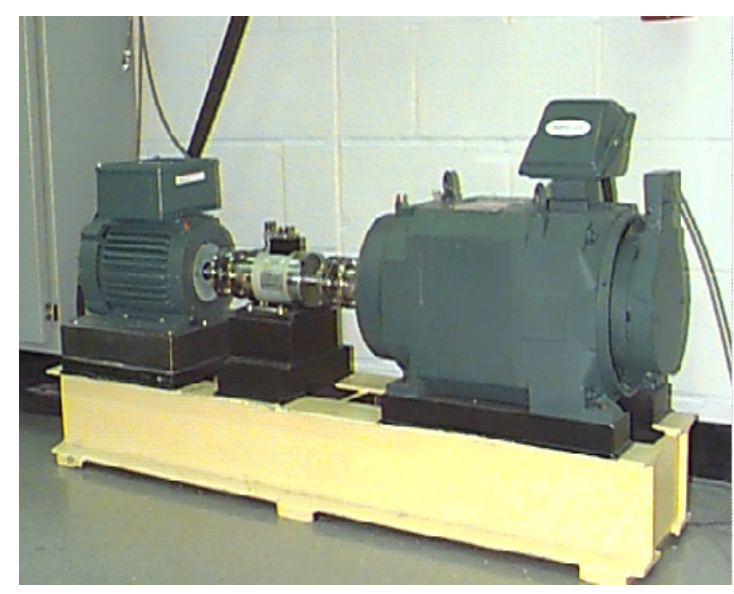

Fig. 5 Experimental device.

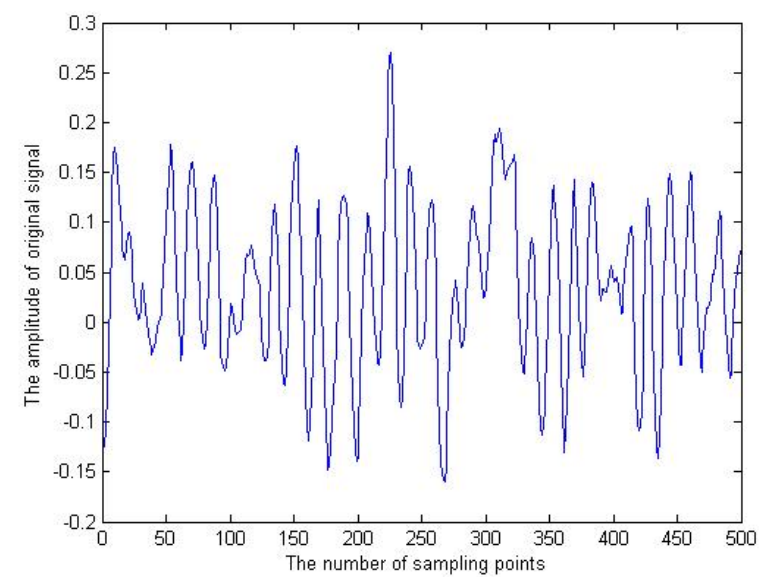

Fig. 6 The part of origin signal.
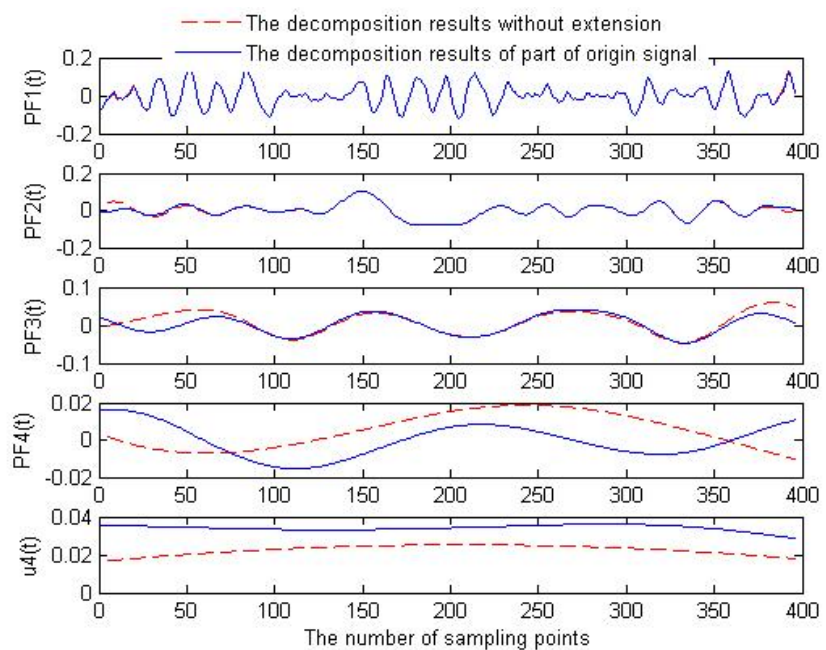

Fig. 7 Decomposition of LMD without extrension and origin signal.

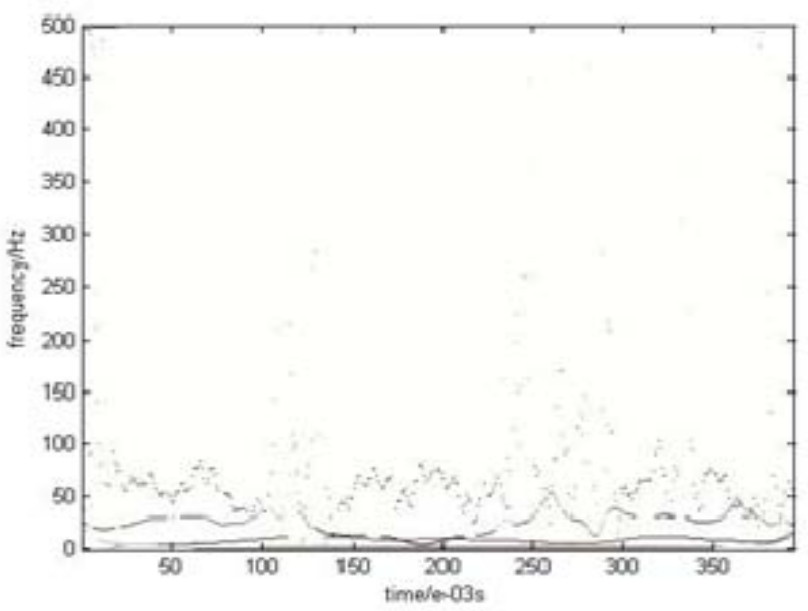

Fig. 8 Hilbert-Huang spectrum without ertension. 


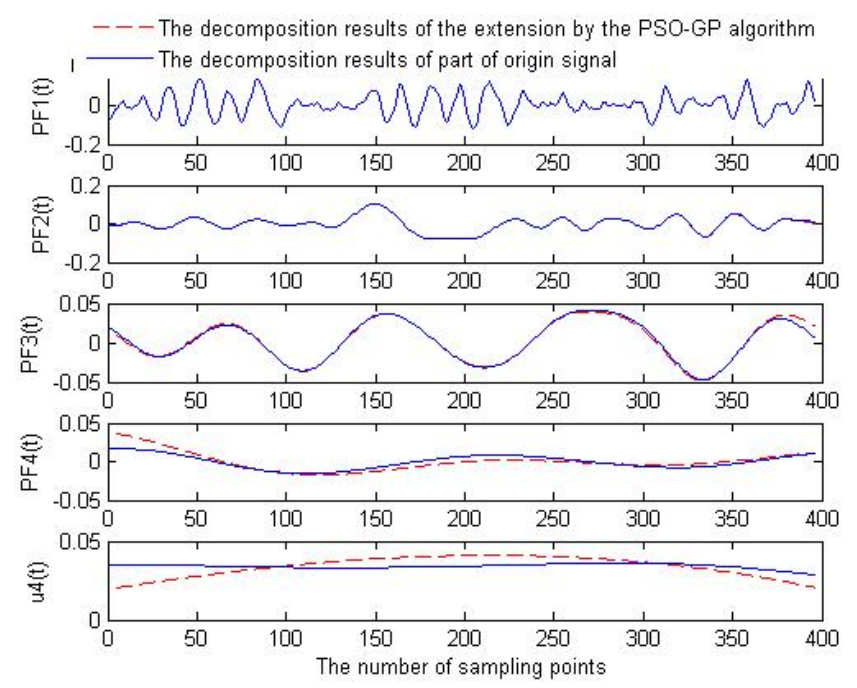

Fig. 9 Decomposition of ertension by PSO-GP algorithm.

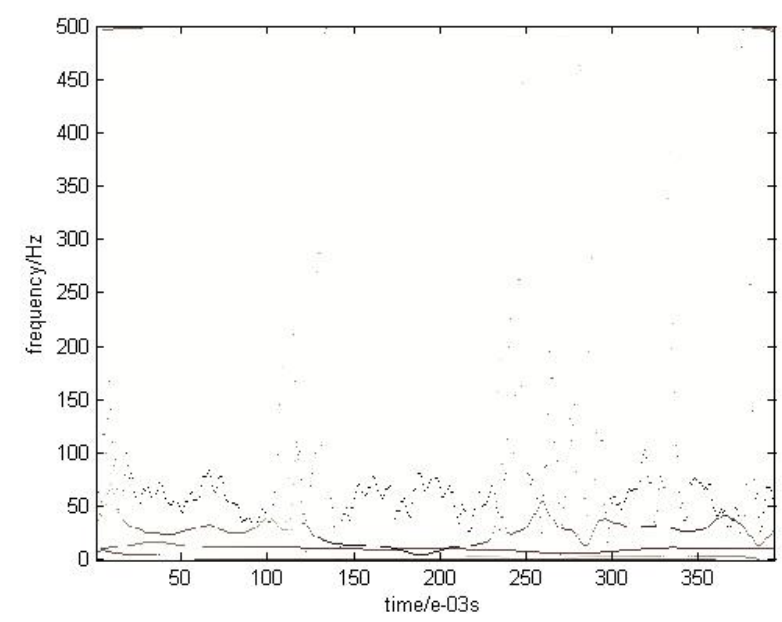

Fig. 10 Hilbert-huang spectrum by PSO-GP algorithm.

From Fig. 7-10, it is clearly shown that by using PSO-GP arithmetic, original column can be effectively continued, thus weakening the adverse effect of end effect on LMD decomposition. The decomposition components attained is compared with the decomposition components of entire signal sequence. Fig. 8 and 10 is the comparison of Hilbert spectrum. As the sample signal sequence is relatively short, the comparison shows no clear result. To further test the level of end effect improvement, two measures are adopted to evaluate the inhibitory effect of factors on end effect. The evaluation result is shown in Table 3.

Table 3. Comparision of methods of end effect.

\begin{tabular}{ccc}
\hline $\begin{array}{c}\text { Evaluation } \\
\text { Index }\end{array}$ & $\begin{array}{c}\text { Without } \\
\text { extension }\end{array}$ & $\begin{array}{c}\text { PSO-GP } \\
\text { method }\end{array}$ \\
\hline$\theta$ & 0.4672 & 0.2755 \\
$\rho_{P F_{1}}$ & 0.9989 & 0.9999 \\
$\rho_{P F_{2}}$ & 0.9714 & 0.9996 \\
$\rho_{P F_{3}}$ & 0.8563 & 0.9941 \\
$\rho_{P F_{4}}$ & 0.1115 & 0.8826 \\
$\rho_{P F_{5}}$ & 0.2821 & 0.3482 \\
\hline
\end{tabular}




\section{Conclusion}

Focused on the problem of LMD's end effect and based on theoretical foundation of LMD, the authors analyzed the reasons for end effect deeply. In view of limitation of traditional endpoint extension under the circumstance of dealing with non-linear and non-stationary signals, the authors consider the high adaptability of Gaussian process when tackling non-linear signals, combined with consideration of hyper parameters having the possibility to be the local optimal value. Then particle swarm algorithm is utilized to optimize hyper parameter, which aims to improve the accuracy of GP algorithms endpoint extension further, as well as apply it in simulation signals and practical mechanical vibration signals. Through theoretical analysis and practical calculation, PSO-GP algorithm can effectively suppress the influence of end effect. Through Hilbert-Huang spectrum and each evaluation index, it is found that based on PSO-GP, LMD decomposition can be a good method of inhibiting end effect during LMD decomposition, which improve the accuracy of LMD decomposition, try its best to preserve the internal characteristics of the signal and avoid end effect polluting internal signal sequences. Therefore this proposal method in this article has good adaptability, as well as excellent ability in normal inhibition of end effect and is easy to calculate and harness.

\section{Acknowledgement}

In this paper, the research was sponsored the National Natural Science Foundation of Chindlda (Project No.41374007) and the Nature Science Foundation of JiangXi Province (Project No. 20151BAB213031) .

\section{References}

[1] J. S. Smith, The Local Mean Decomposition and Its Application to EEG PerceptionData, J. Royal Soc. Interf. 2(5) (2005) 443-454.

[2] N. E. Huang, Z. Shen, S. R. Long, et al. The Empirical Mode Decomposition and the Hilbert Spectrum for Nonlinear and Non-Stationary Time Series Analysis, P. Royal Soc. Lond. A, (1998) 903-995.

[3] N. E. Huang, Z. Shen, S. R. Long,et al. The Empirical Mode Decomposition and the Hilbert Spectrum for Nonlinear and Non-stationary Time Series Analysis. Proc. R. Soc. Lond. A. 454 (1998) 903-995.

[4] N. E. Huang, Z. Shen, S. R. Long, A New View of Nonlinear Water Waves:the Hilbert Spectrum, Annu. Rev. Fluid Mech. 31 (1999) 417-457.

[5] D. M. Klionski, N. I. Oreshko, V. V. Geppener,et al. Applications of empirical mode decomposition for processing nonstationary signals, Pattern Recogn. Image Anal. 18(3) (2008) 390-399.

[6] D. P. Madnic, M. Golz, A. Kuh, et al. Signal processing techniques for knowledge ertraction and information fusion, Berlin: Springer, 2007.

[7] Y. S. Lee, S. Tsakirtzis, A. F. Vakakis, et al. Physics-based foundation for empirical mode decomposition, AIAA J. 47(12) (2009) 2938-2963.

[8] D. J. Huang, J. P. Zhao, J. L. Su, Praetical implementation of the Hilbert-Hunag Trnasofm algorithm, Acta. Oceanol. Sinica. 25(1) (2003) 1-11.

[9] Q. Gai, X. J. Ma, H. Y. Zhang, et al. New method for processing end effect in local wavemethod, J. Dalian U. Technol. 42(1) (2002) 115-117. 
[10]Y. J. Deng, W. Wang, C. C. Qian, et al. Comments and modifieations on EMD method, Chinese Sci. Bulletin. 46(3) (2001) 257-263.

[11]Y. S. Qu, Research on data processing based on Gaussian Process. Beijing Jiaotong U. 2014.

[12]C. E. Rasmussen, C. K. I. Williams, Gaussian Processes for Machine Learning, The MIT Press, 2006.

[13]J. S. Cheng, Y. Yang, D. J. Yu, A new time frequency analysis method-the local mean decomposition method, J. Viberation Shock. 27(S1) (2008) 129-131.

[14]D. Q. Ren, Study on methods and system for fault characteristics ertraction of rotating machines based on local mean decomposition, Hangzhou:ZheJiang U. 2008.

[15]K. Zhang, Research on local mean decomposition method and it's application to rotating machinery fault diagnosis, Changsha:Hunan U. 2012.

[16]K. Zhang, J. S.Cheng, Y. Yang, Processing Method for End Effects of Local Mean Decomposition Based on Self-adaptive Waveform Matching Extending, China Machinical Eng. 21(4) (2010) 457-462.

[17]M. Seeger, Gaussian processes for machine learning, Int. J. Neural. 14(2) (2004) 69-106.

[18] S. U. Guoshao, Forecast of rock burst intensity based on Gaussian process machine learning, J. Liaoning Tech. U. Nat. Sci. 28(5) (2009) 762-765.

[19]B. Sun, H. T. Yao, T. Liu, Short-term Wind Speed Forecasting Based on Gaussian Process Regression Mode, P. CSEE. 32(29) (2012) 104-109.

[20]E. Assareh, M. A. Behrang, M. R. Assari, A. Ghanbarzadeh, Application of PSO (particle swarm optimization) and GA (genetic algorithm) techniques on demand estimation of oil in Iran. Energy, 2010, 3512.

[21] J. X. Li, C. L. Han, C. S. Wang, Z. H. Qie, The TAM and PSO-SVM methods for assessing the crack damage of concrete slab, Optik - Int. J. Light Electron Optics, 2013.

[22]P. J. García Nieto, E. García-Gonzalo, J. R. Alonso Fernández, C. Díaz Muñiz, Hybrid PSO-SVM-based method for long-term forecasting of turbidity in the Nalón river basin: A case study in Northern Spain, Ecol. Eng. 2041, 73.

[23]F. Shi, H. Wang, L. Yu, et al. Thirty case of MATLAB intelligent algorithm, BeiHang U. Press, 2011.

[24]C. Xu, B. G. Liu, K. Y. Liu, et al. Intelligent analysis model of landslide displacement time series based on coupling PSO-GPR, Rock Soil Mech. 32(6) (2011) 1669-1675.

[25]M. Lei, Gaussian process of deformation based algorithm, East China U. Technol. 2013.

[26] V. Vapnik, The Nature of Statistical Learning Theory, 2nd ed. New York:Springer-Verlag, (1998) 360-374.

[27]L. Lin, T. Zhou. L. Yu, Edge Effect Processing Technique in EMD Algorithm, Comput. Eng 35(23) (2009) 265-268.

[28]C. H. Bai, X. H. Zhou. D. C. Lin. et al. PSO-SVM method based on elimination of end effects in EMD, Syst. Eng. Theor. Pract. 5 (2013) 1298-1306.

[29]Z. X. Zhang, Application and design of matlab Programming, Beijing: Press of TsingHua U. 2002. 